\title{
Loss of Flight and Associated Neuronal Rhythmicity in Inositol 1,4,5-Trisphosphate Receptor Mutants of Drosophila
}

\author{
Santanu Banerjee, ${ }^{1}$ Jisue Lee, ${ }^{2} \mathrm{~K}$. Venkatesh, ${ }^{1}$ Chun-Fang Wu, ${ }^{2}$ and Gaiti Hasan ${ }^{1}$ \\ ${ }^{1}$ National Centre for Biological Sciences, Tata Institute of Fundamental Research, Gandhi Krishi Vigyan Kendra Campus, Bangalore 560065, India, and \\ ${ }^{2}$ Department of Biological Sciences, University of Iowa, Iowa City, Iowa 52242
}

Coordinated flight in winged insects requires rhythmic activity of the underlying neural circuit. Here, we show that Drosophila mutants for the inositol 1,4,5-trisphosphate ( $\mathrm{Ins}_{3}$ ) receptor gene (itpr) are flightless. Electrophysiological recordings from thoracic indirect flight muscles show increased spontaneous firing accompanied by a loss of rhythmic flight activity patterns normally generated in response to a gentle puff of air. In contrast, climbing speed, the jump response, and electrical properties of the giant fiber pathway are normal, indicating that general motor coordination and neuronal excitability are much less sensitive to itpr mutations. All mutant phenotypes are rescued by expression of an $\mathrm{itpr}^{+}$transgene in serotonin and dopamine neurons. Pharmacological and immunohistochemical experiments support the idea that the $\mathrm{Ins}_{3}$ receptor functions to modulate flight specifically through serotonergic interneurons. InsP $\mathrm{P}_{3}$ receptor action appears to be important for normal development of the flight circuit and its central pattern generator.

Key words: calcium; aminergic; serotonin; central pattern generator; inositol 1,4,5-triphosphate receptor; flight

\section{Introduction}

The second messenger inositol 1,4,5-trisphosphate $\left(\mathrm{InsP}_{3}\right)$ can be generated in response to a variety of extracellular signals such as hormones, neurotransmitters, and growth factors (Berridge, 1993). Its target within the cell is the Ins $\mathrm{P}_{3}$ receptor $\left(\mathrm{InsP}_{3} \mathrm{R}\right)$, which functions as a ligand-gated channel for the release of calcium from intracellular stores. The physiological role of $\mathrm{InsP}_{3^{-}}$ mediated intracellular calcium release is only just beginning to be understood. Given the widespread tissue expression of the Ins $\mathrm{P}_{3} \mathrm{R}$, it is likely to regulate multiple developmental, physiological, and neuronal signaling pathways (Berridge, 1998).

A consistent problem in studying the role of $\mathrm{Ins}_{3}$ signaling in adults, particularly in the context of the whole organism, has been the absence of viable mutants in the Ins $\mathrm{P}_{3} \mathrm{R}$, presumably resulting from a critical requirement for its function during development. Mutants in genes for the $\mathrm{InsP}_{3} \mathrm{R}$ exist in mouse (Matsumoto et al., 1996), Drosophila melanogaster (Acharya et al., 1997; Venkatesh and Hasan, 1997; Venkatesh et al., 2001), and Caenorhabditis elegans (Clandinin et al., 1998). The mouse $\mathrm{InsP}_{3} \mathrm{R}$ type 1 knockout strain is semilethal. The few surviving mice exhibit ataxia and epileptic behavior. Analysis of cerebellar slices from mutant mice has shown that the $\operatorname{Ins}_{3} \mathrm{R}$ is required for induction of long-term

Received Feb. 24, 2004; revised July 23, 2004; accepted July 28, 2004.

This work was supported by core funding from National Centre for Biological Sciences, Tata Institute of Fundamental Research (TIFR), and a grant from the Department of Science and Technology (India) to G.H. S.B.'s visit to the University of lowa was supported by Travel Fellowships from the Journal of Cell Science and the International Brain Research Organization.

Correspondence should be addressed to Gaiti Hasan, National Centre for Biological Sciences, TIFR, Gandhi Krishi Vigyan Kendra Campus, Bellary Road, Bangalore 560065, India. E-mail: gaiti@ncbs.res.in.

K. Venkatesh's present address: Gene Expression Laboratory, Institute of Ecology, University of Lausanne, $\mathrm{CH}$ 1015 Lausanne, Switzerland

DOI:10.1523/JNEUROSCI.0656-04.2004

Copyright $\odot 2004$ Society for Neuroscience $\quad$ 0270-6474/04/247869-10\$15.00/0 depression (LTD) in Purkinje cells (Miyata et al., 2000). LTD is thought to be one of the cellular correlates of adult motor learning. In C. elegans, the $\mathrm{InsP}_{3} \mathrm{R}$ is directly responsible for maintaining a $50 \mathrm{sec}$ behavioral rhythm for defecation (Dal Santo et al., 1999). These studies demonstrate the relevance of $\mathrm{InsP}_{3}$ signaling in modulating complex adult behaviors and also underline the importance of generating mutant alleles that can be studied as adults. Toward this end, we generated a set of point mutant alleles for the InsP ${ }_{3} \mathrm{R}$ gene (itpr) in Drosophila, some of which are viable as heteroallelic combinations (Deshpande et al., 2000; Joshi et al., 2004). Their viability is very likely attributable to compensatory effects among different mutant monomers, enabling assembly of a functional tetrameric form of the $\operatorname{Ins}_{3} \mathrm{R}$ (Maeda et al., 1991; Serysheva et al., 2003). Single-channel studies with some mutant forms support this idea (Srikanth et al., 2004).

In a previous study, it was shown that viable mutant combinations for the itpr gene are affected in maintenance of olfactory adaptation in the antennae but are normal when tested for primary olfactory transduction (Deshpande et al., 2000). Indeed, visual transduction in Drosophila photoreceptors lacking the itpr gene is also normal (Raghu et al., 2000). To identify neural processes sensitive to InsP ${ }_{3} \mathrm{R}$ activity, motor functions like the jump response, climbing speed, and cleaning reflex were tested in adult viable itpr mutants and found to be unimpaired. In contrast, flight is compromised to various levels in all mutant combinations. Our studies show that flight-related rhythmic activity in the dorsal longitudinal muscles (DLMs) requires itpr gene function in aminergic neurons at the time of pupation and possibly in adulthood. The $\mathrm{InsP}_{3} \mathrm{R}$ most likely modulates the activity of serotonin interneurons, which appear especially vulnerable to itpr mutations. This modulation seems crucial for proper function of the flight central pattern generator. 


\section{Materials and Methods}

Drosophila strains. Different mutant alleles for the itpr gene were used to test for flight. The viable heteroallelic combinations used were wc703/ ug3, ka1091/ug3, sa54/ug3, wc361/ug3, wc703/wc361, sa54/wc361, and $k a 1091 / w c 361$. All of these are single-point mutants in the itpr gene that were generated in an ethylmethane sulfonate (EMS) screen. Detailed molecular information on these alleles has been published recently (Joshi et al., 2004; Srikanth et al., 2004). For all rescue experiments, an embryonic wild-type (WT) itpr cDNA [upstream activation sequence (UAS)$i t \mathrm{pr}^{+}$] was used (Venkatesh and Hasan, 1997). The GAL4 strains used were elavGAL4 (Luo et al., 1994), Dmef2GAL4 (Ranganayakulu et al., 1996), ChATGAL4 (Yasuyama and Salvaterra, 1999), neuralisedGAL4 (Jhaveri et al., 2000), DdcGAL4 (Li et al., 2000), OK6GAL4 (Aberle et al., 2002), Shaking B GAL4 (Jacobs et al., 2000), and two hsp70GAL4 strains referred to as $h_{s} G A L 4$ and $h_{s} G A L 4^{(t)}$, both of which were obtained from the Bloomington Stock center. $h s G A L 4$ showed basal green fluorescent protein (GFP) expression at $25^{\circ}$ when crossed to UAS-GFP, whereas $h s G A L 4^{(t)}$ did not show any GFP expression under the same experimental conditions.

Heat shock experiments. For the experiments in Figures 1-3, flies with the $h s G A L 4$ transgene were maintained throughout at $25^{\circ} \mathrm{C}$. Basal level of GAL4 expression at this temperature was sufficient to rescue all of the phenotypes described. For experiments with the $h s G A L 4(t)$ transgene (see Fig. 7), animals of the appropriate genotype were raised at $25^{\circ} \mathrm{C}$ throughout development. In the designated cases, a heat shock of $37^{\circ} \mathrm{C}$ was given for $1 \mathrm{hr}$ to pupae of the denoted age ranging from 16 to $24 \mathrm{hr}$ after puparium formation (APF) to $80-88 \mathrm{hr}$ APF. In addition, a $1 \mathrm{hr}$ heat shock at $37^{\circ} \mathrm{C}$ was given to adult flies of the genotype UAS-itpr ${ }^{+/+}$; hsGAL4 $4^{(t)} /+$; neurGAL4, itpr $r^{u g 3} /$ itpr $^{w c 703}$. These animals are referred to as adults (neur) in Figure 7A. For experiments in Figure 7B, a $1 \mathrm{hr}$ heat shock of $37^{\circ} \mathrm{C}$ was delivered to vials containing flies of various developmental stages. Each following day, newly emerged flies were collected for examination. Thus, flies received heat shock at different developmental stages, determined by the time lapse between heat shock and eclosion, could be obtained from the same treatment. Before electrophysiological recordings, flies were randomly selected, tethered to a mounting wire, and tested for air puff-induced tethered flight, which mimics free flight. Flies are grouped according to early (first $2 \mathrm{~d}$ ) and late (last $2 \mathrm{~d}$ ) pupal, adult (first day) heat shock, and no heat shock.

Flight assay. Flight tests were performed following minor modifications of the "cylinder drop assay" described previously (Benzer, 1973). A batch of 20 flies was tested each time. A minimum of five batches was tested for each genotype (except in Figs. $6 \mathrm{~A}$ and $7 \mathrm{~A}$; see respective figure legends for details). Flies that dropped directly down the cylinder were collected in a vial kept on ice under the bottom opening of the cylinder. These were counted as defective in flight. Flies that were able to hold onto the walls of the cylinder were considered fliers. The percentage of flight defective organisms was determined as: number of flies dropped in the vial/total number of flies tested $\times 100$.

Electrophysiological preparation and recordings. Physiological recordings were performed on the DLMs of the giant fiber (GF) pathway (Tanouye and Wyman, 1980). Recording methods for the muscle have been described previously (Engel and Wu, 1992; Lee and Wu, 2002). Flies were briefly etherized and glued to a metal wire between the neck and thorax using nail polish. Tungsten electrodes were used for stimulation and recording. A recording electrode was inserted into a DLM in the thorax, and a reference electrode into the abdomen. The recording electrode penetrated the scutum into the most dorsal DLM muscle (fiber a) on the right or left side. Although electrodes were uninsulated, only the tips of recording electrodes were inserted into the muscle that lie just beneath the cuticle; thus, influence from neighboring muscle fibers was negligible. Flies were rested at least $30 \mathrm{~min}$ before recordings. During the recording of spontaneous activities, the fly was kept undisturbed in the chamber covered with a dark screen. The fly was blown on (air puff) to induce flight. To determine the GF pathway response latency, stimuli $(0.1 \mathrm{msec})$ from a stimulator (Grass SD9 with internal SIU; Grass Instruments, Quincy, MA) were passed via two tungsten electrodes inserted into the eyes. Usually, a relatively weak stimulus produced a long latency response, and higher stimulus voltages produced a short latency response (Engel and Wu, 1992). Response latency was defined as the interval between the stimulus artifact and the final inflection of the rising phase of the muscle action potential. Responses were picked up with an AC preamplifier (filter bandwidth from $0.1 \mathrm{~Hz}$ to $>30 \mathrm{kHz}$, reference grounded; DAM-5A; World Precision Instruments, New Haven, CT) and recorded with pulse code modulation (DR-384; NeuroData, New York, NY) on videotapes at a sampling rate of $44 \mathrm{kHz}$. Gap-free recording mode of fetchex in pClamp6 (Axon Instruments, Foster City, CA) was used for digital data acquisition $(1 \mathrm{kHz})$ on a Pentium computer equipped with Digidata 1200 (Axon Instruments). Spikes were detected using Axograph 3.0 (Axon Instruments). All physiological experiments were performed at room temperature $\left(22-27^{\circ} \mathrm{C}\right)$.

Statistical analysis. For all behavioral experiments (flight assay), at least 100 flies were tested for all genotypes in batches of 20. The mean was calculated from five such trials and plotted with SD using Sigma (St. Louis, MO) Plot software. In Figures $6 A$ and $7 A$, a minimum of six flight tests were done for each time point, genotype, or treatment, with batches of 10 flies in each case. The data were analyzed as described above. In Figure 2, the number of spontaneous spikes was obtained from each fly in a random sample of $2 \mathrm{~min}$. Mean and SD computation and $t$ tests were performed using Microsoft Excel software.

Drug-feeding experiments. 3-iodotyrosine (3-IY; $10 \mathrm{mg} / \mathrm{ml}$ ) or parachlorophenylalanine (PCPA; $10 \mathrm{mg} / \mathrm{ml}$ ) were dissolved in $5 \%$ sucrose plus $2 \%$ yeast solution and fed to adult flies as a freshly made solution for each of $5 \mathrm{~d}$. On the fifth day, flight tests were performed as described above.

Immunohistochemistry and microscopy. OK6GAL4 flies were mated to a UAS-GFP strain to visualize motor neurons. In the adult CNS, the expression of OK6GAL4 is not limited to motor neurons. Some expression is observed in cell bodies that lie in the optic lobe (Fig. 8). To visualize serotonergic projections, a monoclonal anti-5-HT antibody (NeoMarkers, Fremont, CA) was used at a dilution of 1:50 and subsequently observed with an anti-mouse Alexa red (Molecular Probes, Eugene, OR) secondary, used at a dilution of 1:400. Flies were $3-5 \mathrm{~d}$ of age at the time of anti 5-HT staining. All imaging was done using either $10 \times 0.3$ numerical aperture (NA) or $60 \times 1.4$ NA objectives on a Bio-Rad (Hercules, CA) MRC 1024 confocal microscope. The appropriate number of sections of $0.3 \mu \mathrm{m}(60 \times)$ or $1.3 \mu \mathrm{m}(10 \times)$ were merged to reveal neuronal trajectories.

\section{Results}

\section{Flight deficits in itpr mutants}

Several single-point mutations in the itpr locus were obtained in a recent mutagenesis. Although the majority of these are homozygous lethal, heteroallelic combinations of certain hypomorphic alleles survive to adulthood (Joshi et al., 2004). These adults appear developmentally normal, but some combinations show a defective wing posture (Fig. 1A) [compare with Canton S (CS) wild type], which is similar to the previously described phenotype of "down-turned" wings (Engel and Wu, 1992; Huang and Stern, 2002). This phenotype is strongly penetrant in all animals, with one copy of $i t p r^{u g 3}$ and one copy of another itpr allele such as $i t p r^{w c 703}, i_{p} r^{k a 1091}$, or $i t p r^{s a 54}$. In addition, itpr ${ }^{\text {sa54 } / i t p r^{u g 3}}$ (sa54/ ug3 for short) heteroalleles exhibit a partially penetrant phenotype of crumpled wing margins (Fig. $1 A$ ). To establish that altered wing posture is a specific effect of mutation of the itpr locus, a UAS-itpr ${ }^{+}$cDNA transgene (Venkatesh et al., 2001) was expressed in the mutants using a ubiquitous hsp70GAL4 (hsGAL4) driver. Flies of the genotype UAS-itpr ${ }^{+/+}$; hsGAL4/+; itpr ${ }^{E M S 1}$ / $i_{\text {tpr }}^{E M S 2}$ (when grown at $25^{\circ} \mathrm{C}$ to allow basal level of $h s G A L 4$ expression) exhibit normal wing posture (Fig. $1 A$ ).

The down-turned wing posture of itpr mutants prompted us to examine their flight behavior. Adult viable itpr mutant combinations were examined for their ability to fly in a behavior test, which is a modification of the "cylinder drop test" (Benzer, 1973) (see Materials and Methods). Flight defects ranging from 30 to 
A
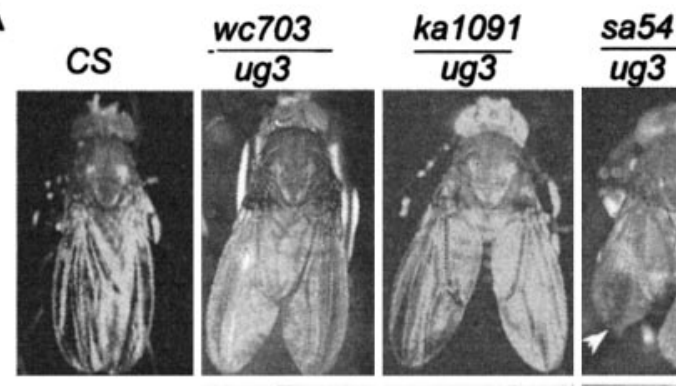

UAS-itpr, hsGAL4/+
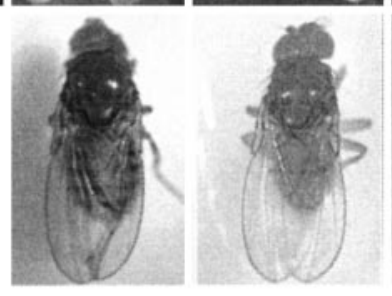

B

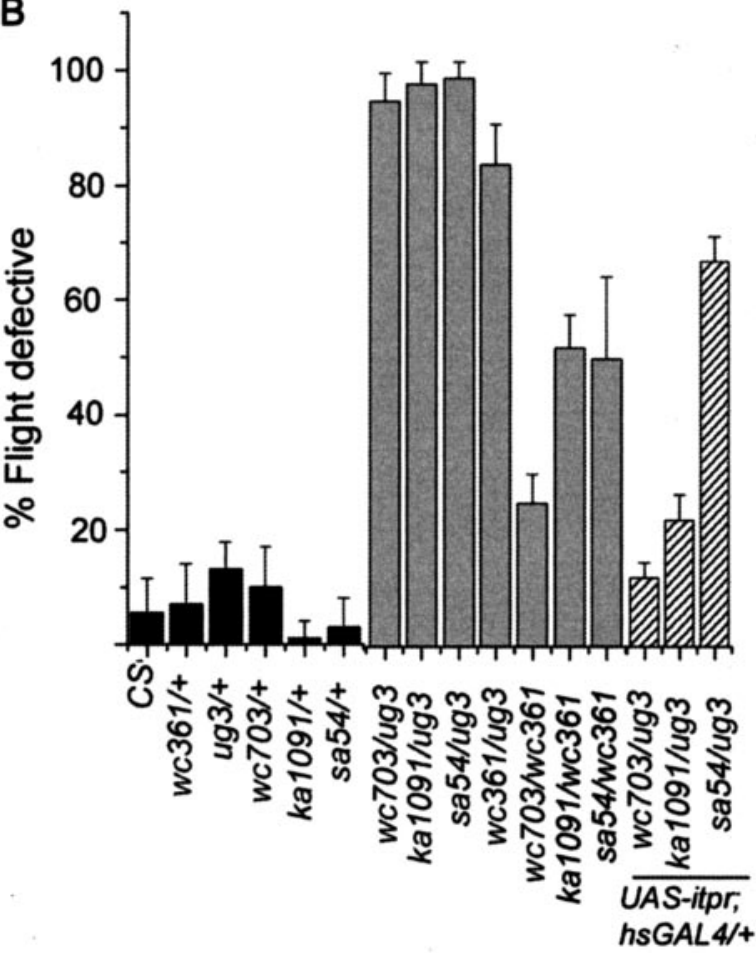

Figure 1. Altered wing posture and flight in viable itpr heteroallelic combinations and their rescue by a UAS-itpr ${ }^{+}$transgene. A, Heteroallelic genotypes (itpr ${ }^{E M S 1} /$ itpr $^{E M S 2}$ ) have been designated by names of the respective alleles (ka1091, wc703, ug3, and sa54). Wild-type flies (CS) exhibit normal wing posture. In wc703/ug3 and ka1091/ug3 organisms, the wing posture is altered and is similar to down-turned wings described previously (Huang and Stern, 2002). Wing posture phenotype is $100 \%$ penetrant for the heteroallelic combinations shown. sa54/ ug3 organisms have an altered wing posture and crumpled wing margins (arrowhead). hsGAL4 (single copy on second chromosome) driven basal expression of an UAS-itpr ${ }^{+}$transgene (single copy on X chromosome/) (Venkatesh et al., 2001) at $25^{\circ}$ ( rescues the wing posture in all mutant genotypes tested. A strict quantification of the wing phenotype was not possible because it cannot be distinguished reliably in anesthetized flies. $B$, Genotypes tested for flight are indicated under each bar in the graph. Flight behavior of itp $^{E M S} /{ }^{+}$flies is not significantly different from that of wild-type flies (CS; black bars). All wc361/itpr ${ }^{E M S}$ combinations have flight defects ranging from 30 to $65 \%$ (gray bars). These combinations did not show any wing posture defects (see Results). Combinations of ug3/itpr ${ }^{E M S}$ have severe flight defects ranging from 80 to $98 \%$ (gray bars) and defects in wing posture $(A)$. These flight defects can be rescued by ubiquitous expression of a UAS-itpr ${ }^{+}$transgene (hatched bars). An hsGAL4 promoter construct on the second chromosome was used to drive expression of one copy of $\mathrm{UAS}^{-i t p r}{ }^{+}$on the $\mathrm{X}$ chromosome in otherwise mutant backgrounds. In wc703/ug3 and ka1091/ug3 organisms, flight defects were rescued completely, whereas in sa54/ug3 flies, a limited rescue of flight defects was seen (see Results for details).
98\% were observed in different heteroallelic combinations (Fig. $1 B$ ). All heteroallelic combinations with $u g 3$ showed severe flight defects (>90\%; except wc361/ug3). Interestingly, heteroallelic mutant combinations with wc361 did not show altered wing posture (data not shown) but did show a significant degree of flightless behavior. Defects in flight in the itpr mutants were recessive (Fig. $1 B$ ). As in the case of the wing phenotype, flight could be rescued by ubiquitous expression of UAS-itpr ${ }^{+}$under control of the hsGAL4 driver in otherwise mutant backgrounds. Flight defects of two heteroallelic combinations were rescued to $80 \%$ (ka1091/ug3) and 90\% (wc703/ug3). In sa54/ug3 organisms, the rescue was significant (30\%) but lower than the other two combinations. It was noted that a number of sa54/ug3 animals expressing the $\mathrm{itpr}^{+}$transgene still showed crumpled wing margins. In the "flier" population of the above genotype, both wing morphology and posture were rescued. Thus mutations in the itpr gene directly affect adult wing posture and flight behavior. These phenotypes could also arise as a consequence of general defects in motor coordination. Therefore, we tested the motor response of itpr mutants in tests that measure climbing rate (Bainton et al., 2000), the jump response (Ikeda and Koenig, 1988a), and the cleaning reflex (Vandervorst and Ghysen, 1980; Burg and Wu, 1986). In all cases, the responses obtained were indistinguishable from wild type (data not shown). The observed changes in wing posture and flight thus arise from specific $\operatorname{defect}(\mathrm{s})$, which could be an alteration in indirect flight muscle function in turn caused by developmental and/or physiological changes (Fleming et al., 1989; DeSimone and White, 1993). The physiological changes could lie in the muscle or in any part of a neural circuit for flight. No obvious developmental defects were observed in the indirect flight muscles either by light or electron microscopy (data not shown), which prompted us to look at the physiology of flight in these mutants.

\section{Altered electrophysiological responses from DLMs of itpr mutants}

To investigate flight physiology in itpr mutants, electrophysiological responses from the dorsal longitudinal set of indirect flight muscles (DLMs) were recorded. The contraction of these muscles powers the upstroke-downstroke of the wing (wing depressor) during flight. Responses from these muscles can serve as a read out of the entire flight circuit and the giant fiber pathway, which consists of sensory inputs, followed by CNS processing for flight pattern generation and leading to motoneuron excitation. Initially, spontaneous discharges were recorded from the DLMs in the absence of any electrical or sensory stimulation. Interestingly, a significantly higher level of spontaneous firing was observed in itpr mutants, compared with wild-type controls (Fig. 2A). Wildtype animals show mostly no bursting activity period with occasional spike discharges, so that an average spontaneous spike frequency of $\sim 2 \mathrm{~Hz}$ was observed during the recording period (Fig. $2 B$ ). The behavioral correlate of these spontaneous brief bursts in wild type is thought to be wing movements associated with cleaning activity (Lee and $\mathrm{Wu}, 2002$ ). In contrast, itpr mutant animals show periods of no activity, followed by high electrical activity phases that were characterized by bursts of high-frequency action potentials (Fig. 2A). The periodicity of these no activity-high activity cycles remains relatively constant in both mutant combinations tested (Fig. 2B). These bursts of high-frequency spike activity do not correlate with increased frequency of cleaning behavior. In organisms with basal expression of UAS-itpr ${ }^{+}$under hsGAL4 control, these bursts of spontaneous firing were restored back to wild-type levels (Fig. $2 A, B$ ). 


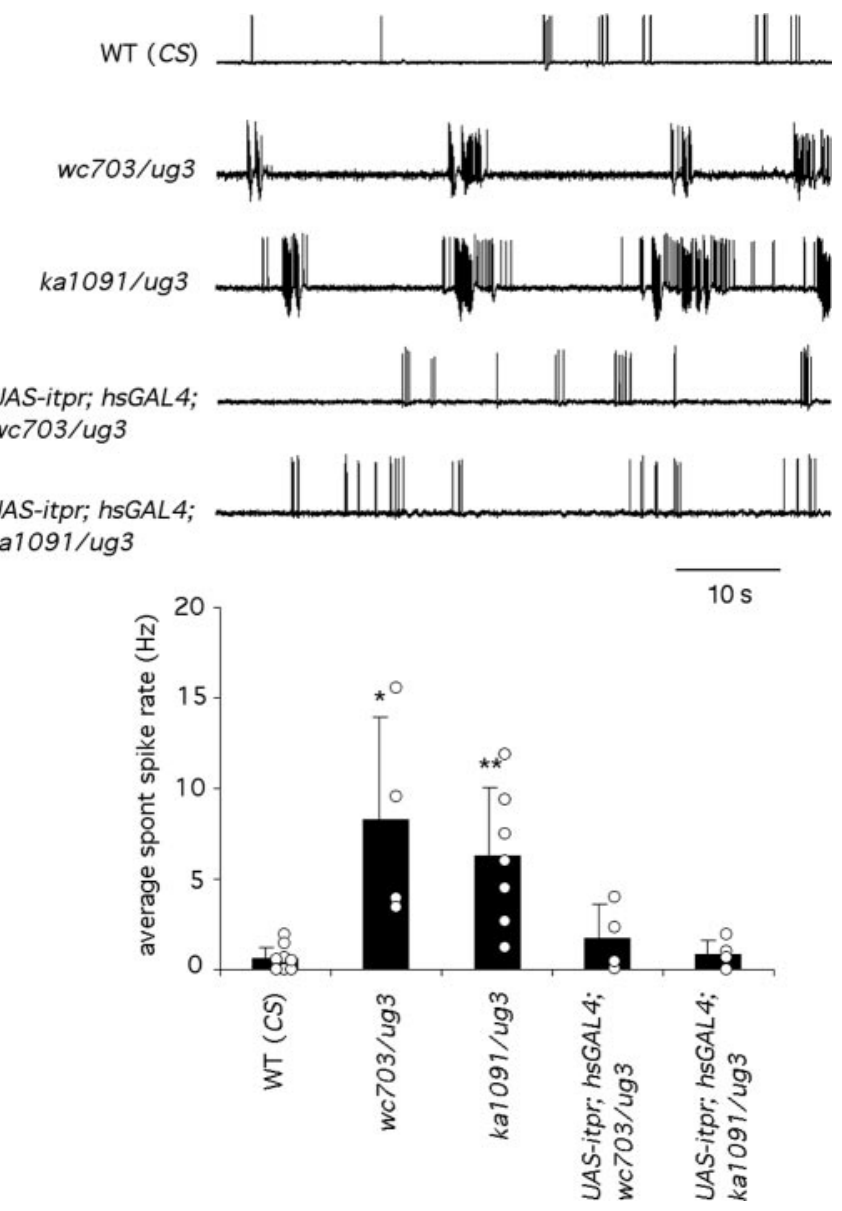

Figure 2. DLMs of itpr mutants exhibit increased levels of spontaneous firing. $A$, DLMs of wild-type flies (CS) can express short bursts of electrical activity in the absence of either mechanical or electrical stimulation. These spontaneous bursts of action potentials are unrelated to flight and are usually associated with cleaning behavior in wild-type flies (Lee and Wu, 2002). The DLMs of itpr heteroalleles, wc703/ug3 and ka1091/ug3, exhibit an increased frequency of spontaneous firing, which is unrelated to cleaning behavior. This increased frequency was suppressed by expression of UAS-itpr ${ }^{+}$with hsGAL4 (UAS-itpr ${ }^{+}$; hsGal4; wc703/ug3 in 5 of 5 animals and UAS-itpr ${ }^{+}$; hsGal4; ka1091/ug3 in 6 of 6 animals). DLM activity was recorded in tethered flies that were placed in a dark chamber and kept undisturbed during recording. $B$, Average number of spontaneous spikes recorded from flies of the indicated genotypes. Spontaneous activity was determined by counting the number of spikes that occurred over 2 min. Results from each fly are indicated by open circles. The filled bars indicate the average for all flies of the specific genotype, and error bars indicate SD $\left({ }^{*} p<0.05\right.$; ${ }^{* *} p<0.01$ ).

Changes in synaptic function might underlie the increased frequency of spontaneous firing. To test this, and to examine the reason for flightless behavior in itpr mutants, properties of the GF pathway were investigated. This circuit triggers the jump-andflight response and can be stimulated by either a bright flash of light or a voltage pulse applied across the eyes. Synaptic function of the circuit is measured by the latency periods observed between stimulus and response of the DLM (Tanouye and Wyman, 1980; Engel and Wu, 1996). A relatively weak stimulus recruits GF afferents and produces a long latency response, whereas short latency responses are obtained at higher stimulus intensities that directly activate the giant fiber. As shown in Table 1, the latency periods for long and short latency responses, as well as the threshold voltages required to elicit these responses, were unchanged between itpr mutants and wild-type flies. In addition, the refractory period, which is the shortest interpulse interval in which the muscle can respond to the second stimulus in a "twin pulse stimulation protocol," was also unaltered in itpr mutant flies. These data show that all synapses of the GF pathway, including the neuromuscular junction and muscle excitability, appear functionally intact in $\mathrm{InsP}_{3}$ receptor mutants.

Insect flight can be initiated by a variety of sensory stimuli. Two stimuli that can independently induce flight in experimental conditions are flashes of light and air puffs (Engel and Wu, 1992). These resemble natural environmental cues perceived by the fly. In previous experiments, it has been shown that the delivery of an air puff to the adult fly elicits a typical firing pattern (CS; five of five) associated with flight behavior in the DLMs (Engel and $\mathrm{Wu}$, 1992). Wild-type and itpr mutant animals were subjected to air puff stimulation and the resulting firing pattern recorded from the DLMs (Fig. $3 A$ ). Significantly, $\mathrm{Ins}_{3} \mathrm{R}$ mutants failed to produce DLM flight patterns in response to an air puff ( wc703/ug3, four of four; ka1091/ug3, four of four). In contrast, responses similar to that of wild-type flies were obtained in mutants on expression of the $i_{p r^{+}}$cDNA under control of $h s G A L 4$ (Fig. 3A). Moreover, in rescued flies, the spike frequency of the flight pattern recorded from the DLMs is in the range of 5 to $10 \mathrm{~Hz}(n=4$ for both UAS-itpr ${ }^{+}$; hsGAL4/+; ka1091/ug3 and UAS-itpr ${ }^{+}$; hsGAL4/+; wc703/ug3), similar to that seen in wild-type flies. It should be noted that spontaneous flight activity could be observed in tethered wild-type flies but regular firing, at a flight frequency, was never observed in itpr mutant flies. These data suggest a physiological basis for why itpr mutants fail the cylinder drop test.

\section{Flight defects in itpr mutants are not attributable to altered wing posture}

Although rescue of itpr mutant phenotypes by expression of the $U A S$-itpr $r^{+}$transgene under control of the $h s G A L 4$ driver clearly demonstrates that these phenotypes are a consequence of itpr gene function, it does not specify the cellular context in which the Ins $\mathrm{P}_{3} \mathrm{R}$ is required during flight. To address this question, we tested several tissue-specific drivers for rescue of $i t p r$ phenotypes. Mutant heteroalleles, which express $U A S-i t p r^{+}$in the domain of neurGAL4 (Jhaveri et al., 2000), exhibit normal wing positions (Fig. 4A). Despite this fact, neurGAL4 rescued flies are unable to fly. This was true for flies expressing either one or two copies of the $i t p r^{+}$transgene (Fig. 4B), indicating that the level of $\mathrm{itpr}^{+}$ cDNA expression is not a factor for the rescue and that rescue of the wing defect is insufficient for rescue of flight in itpr mutants. This idea is further supported by the observation that heteroalleles of $w c 361$ have normal wings (data not shown) but still exhibit varying levels of flight defects (Fig. $1 B$ ). Similarly, rescue of wing position is insufficient for rescuing defects of spontaneous firing and air puff-induced flight as shown for $U A S-i t r^{+/+}$; ug3, neurGAL4/wc703 $(n=5)$ in Figure 5A. Because neurGAL4 expression is predominantly at larval and pupal stages in neuroblasts and

Table 1. Properties of the giant fiber pathway in wild-type and $\ln s \mathrm{P}_{3} \mathrm{R}$ mutant adults

\begin{tabular}{|c|c|c|c|c|}
\hline Genotype $(n)$ & Short latency threshold (V) & Long latency threshold (V) & Short latency refractory period (msec) & Long latency refractory period (msec) \\
\hline CS (WT) (10) & $6.7 \pm 0.6$ & $3.7 \pm 0.6$ & $3.3 \pm 0.6$ & $61.8 \pm 4.3$ \\
\hline Itpr ${ }^{k a 1091} /$ itpr $^{\mu g 3}$ (10) & $6.8 \pm 0.8$ & $3.5 \pm 0.6$ & $3.4 \pm 0.8$ & $57.6 \pm 5.6$ \\
\hline
\end{tabular}




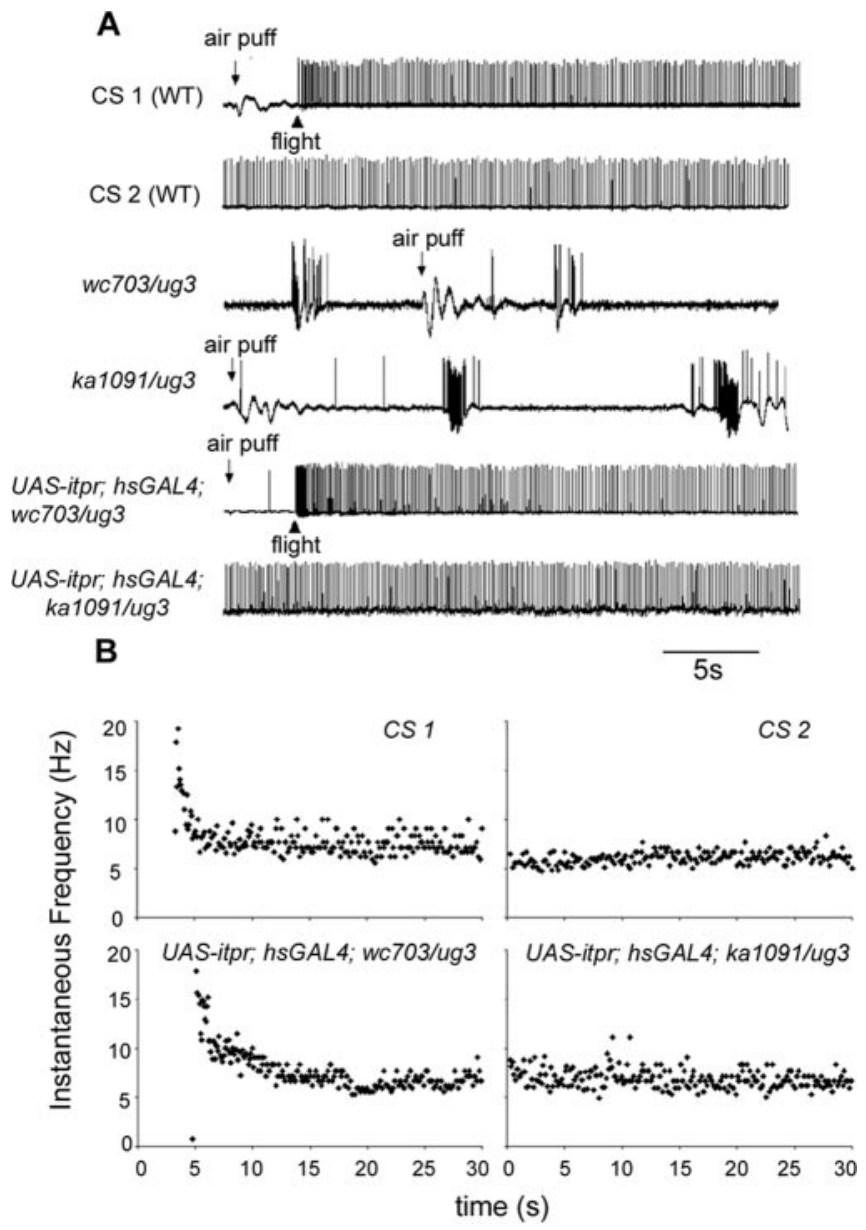

Figure 3. Air puff-induced flight activity is absent in itpr heteroalleles. $A$, Flight induction by an air puff in wild-type (CS) flies as a consequence of which the DLMs exhibit a tonic firing pattern (CS1), which is maintained until cessation of flight (CS2). This flight pattern is distinct from spontaneous spiking activities (Fig. 2), and the two tend to be nonoverlapping. Air puff stimulation failed to induce a flight activity pattern in the DLMs of itpr heteroalleles (wC703/ug3 and ka1091/ug3; 4 of 4 animals that were tested for each genotype) and did not interrupt the aberrant background spontaneous activities. Flight defects in $\operatorname{Ins}_{3} \mathrm{R}$ mutants were restored by the expression of the itpr ${ }^{+}{ }^{C D N A}$ with the hsGAL4 driver (UAS-itpr ${ }^{+}$; hsGal4; wC703/ug3; 4 of 5 animals and UAS-itpr ${ }^{+}$; hsGal4; ka1091/ug3, 4 of 6 animals). B, Expression of the itpr ${ }^{+}$cDNA restores normal frequency of tonic firing in itpr mutants. Examples of flight pattern shown in $A$ have been plotted to show an instantaneous frequency (inversion of interspike interval) pattern. Note that the instantaneous spike frequency is high at the onset of flight and then maintained between 5 and $10 \mathrm{~Hz}$.

developing muscles (Jhaveri et al., 2000), we attributed the wing posture phenotype to a requirement for the $\operatorname{Ins}_{3} \mathrm{R}$ in either one or both of these developing tissues. To distinguish between them, the Dmef2GAL4 driver (Ranganayakulu et al., 1996; Greene et al., 2003), which controls expression in all developing muscles, was used to drive $U A S$-itpr ${ }^{+}$expression. However, no rescue of wing position was observed (Fig. $4 A$ ). Thus, rescue of the wing phenotype appears to require Ins $\mathrm{P}_{3} \mathrm{R}$ function in neuroblasts and possibly their neuronal progeny. This was further corroborated by experiments described below.

\section{The focus of the flight defect in itpr mutants lies in} aminergic interneurons

The absence of rescue of flight and associated physiological defects by neurGAL4 might be caused by its diminished expression in mature neurons. Thus, we tested several GAL4 strains that are known to express in nonoverlapping subsets of adult neurons.
A

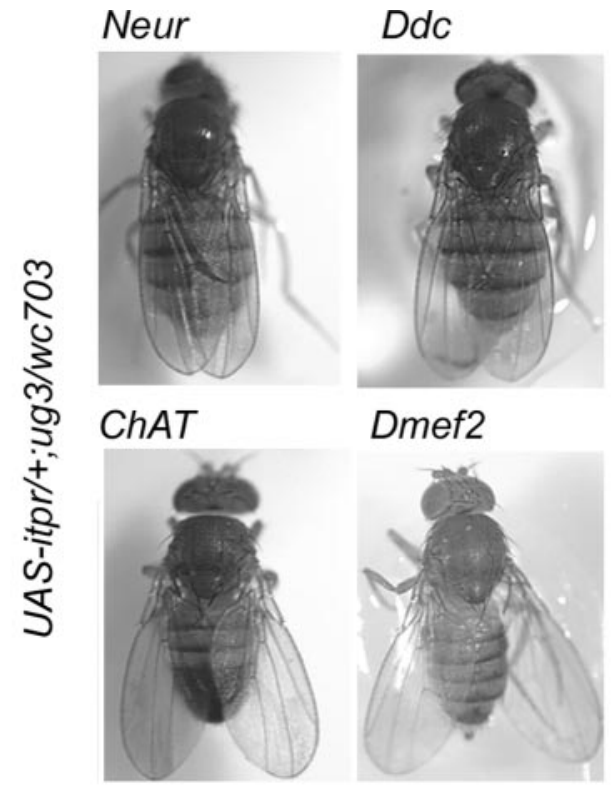

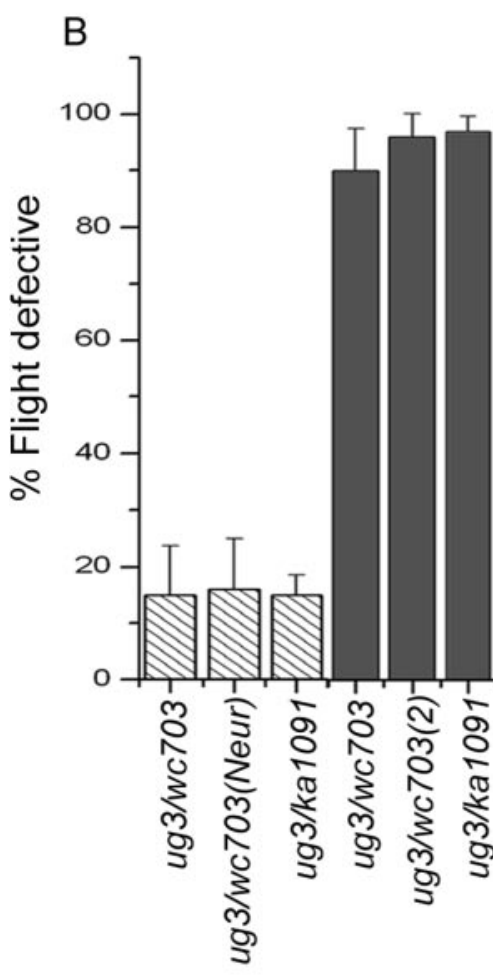

DdcGAL4

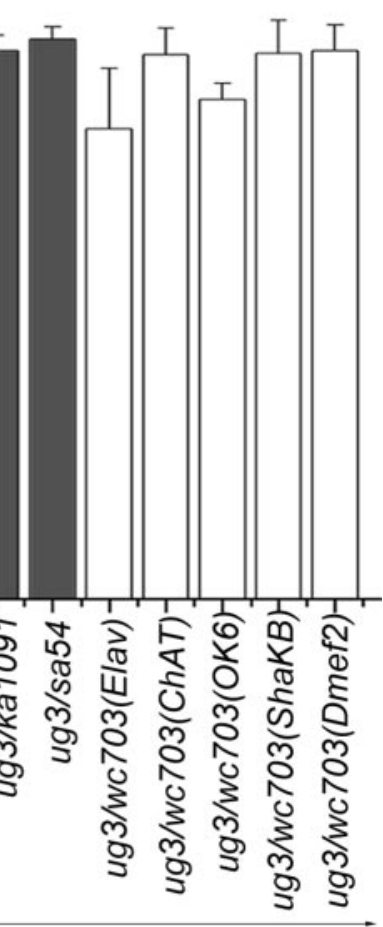

NeurGAL4
Figure 4. Tissue and cell type-specific rescue of wing posture and flight defects in itpr heteroallelic combinations. A, Rescue of wing posture. Expression of UAS-itpr with neurGAL4 and DdcGAL4 rescues wing posture in wc703/ug3, ka1091/ug3 organisms. No rescue was observed when $U A S-i t p r{ }^{+}$expression was driven by GAL4 strains that express in all cholinergic neurons (ChATGAL4) or in all developing muscles (Dmef2GAL4) in wC703/ug3 mutant organisms. B, Rescue of flight behavior. DdcGAL4-mediated expression of UAS-itpr ${ }^{+}$rescues the flight defect close to wild-type levels in itpr mutants (hatched columns). NeurGAL4-mediated expression of one copy of UAS-itpr ${ }^{+}$failed to rescue flight in any combination tested (gray bars). Two copies of UAS-itpr ${ }^{+}$in ug3/wC703(2) organisms still had no effect. Expression of UAS-itpr ${ }^{+}$with elavGAL4 (pan-neural; has uneven levels of expression in adult neurons), ChATGAL4 (cholinergic neurons), Ok6GAL4 (motor neurons), and ShakBGAL4 (giant fiber pathway neurons), in combination with neurGAL4, failed to rescue flight to any significant level (white columns). NeurGAL4 was included for rescue of wing posture. 
A
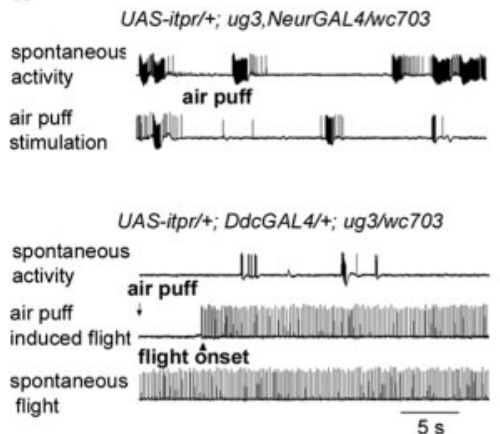

B

UAS-itpr/4; DdcGAL4/+; ug3/wc703

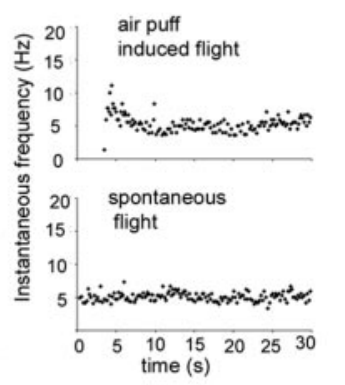

Figure 5. Cell type-specific rescue of increased spontaneous firing and air puff responses. $A$, Expression of UAS-itpr ${ }^{+}$in the domain of the neurGal4 driver (UAS-itpr ${ }^{+/+} ;{ }^{+/+}$; neurGal4, ug3/wc703; 4 of 4 animals) failed to rescue either increased levels of spontaneous firing or the absence of air puff-evoked firing in the DLMs. However, DdcGAL4 driven expression of UASitpr $^{+}$in an itpr mutant combination (UAS-itpr ${ }^{+/+}$;DdcGal4/+; wC703/ug3) suppressed spontaneous firing (5 of 7 animals; compare with WT trace in Fig. 2) and restored normal flight activity patterns (CS1 and CS2 traces in Fig. 3) in response to air puff stimulation. B, Frequency of the firing pattern recorded from DLMs of UAS-itpr ${ }^{+/+} ;$DdcGAL4 rescued itpr mutant flies is similar to that of wild-type flies $(5-10 \mathrm{~Hz}$ ) (Fig. 3). Firing frequency during spontaneous flight, in the rescued strain, is also similar to wild-type levels.

Among these, DdcGAL4 driven expression of UAS-itpr ${ }^{+}$could rescue flight to significant levels (Fig. $4 B$ ). Significantly, both spontaneous firing and air puff-induced flight activity also returned to wild-type levels in these flies $(n=5)$, as shown in Figure $5 B$. The domain of $D d c G A L 4$ expression is in all dopamine and serotonin neurons ( $\mathrm{Li}$ et al., 2000), suggesting their major contribution to the observed rescue. Consistent with this is the observation that no rescue of flight was observed when the $i t p r^{+}$ transgene was expressed in other neuronal subsets such as cholinergic sensory and interneurons (ChATGAL4) (Yasuyama and Salvaterra, 1999), motor neurons (OK6GAL4) (Aberle et al., 2002), and the GF pathway (ShaKBGAL4) (Jacobs et al., 2000). None of these strains were able to rescue altered wing position. Moreover, organisms expressing the UAS-itpr ${ }^{+}$ transgene in these GAL4 domains still failed the flight test, even after restoration of wing position when combined with neurGAL4 (Fig. 4B). In contrast, DdcGAL4 could rescue wing position, flight, and flight physiology, suggesting that the focus of all of these defects lies in aminergic cells (Figs. 4, 5). However, it is important to note that neurGAL4 rescued only the wing phenotype but not flight (Fig. $4 B$ ) and flight physiology (Fig. 5A), indicating that different subsets of aminergic cells contribute to the two phenotypes.

Somewhat surprisingly, no rescue of flight was observed with the pan-neuronal elavGAL4 driver. We attribute this failure to insufficient levels of elavGAL4 driven $U A S$-itpr ${ }^{+}$expression in the requisite cells. Absence of rescue of adult neuronal phenotypes by elavGAL4 driven expres- sion has been noted previously (Zars et al., 2000; Greeve et al., 2004) and in our laboratory for adult olfactory phenotypes associated with the itpr mutants (Deshpande et al., 2000) (data not shown). However, the olfactory phenotype was rescued by ChATGAL4 driven expression of UAS-itpr ${ }^{+}$(data not shown). An alternate reason for absence of rescue by elavGAL4 is that rescue by DdCGAL4 is from epidermal cells that are also known to express dopa decarboxylase (Kraminsky et al., 1980). However, this seems unlikely, primarily because the rescue of electrophysiological defects (as observed in Fig. 5) is unprecedented from epidermal cells and is more likely to occur from neurons.

To assess independently whether evoked release of neurotransmitters from $D d c G A L 4$ neurons is required for flight, a tetanus toxin transgene (UAS-TNT) was used. Expression of tetanus toxin (TNT) in neurons is known to abolish evoked neurotransmitter release and reduce the frequency of spontaneous quantal release (Sweeney et al., 1995). A flight defect of $\sim 45 \%$ was observed in DdcGAL4/UAS-TNT adults (Fig. 6A). Physiological recordings confirmed the important role of $D d c$ expressing neurons in the control of flight pattern generation (Fig. $6 B$ ). Fliers and nonfliers were collected from DdCGAL4/UAS-TNT adults for physiological examinations. Three of three nonfliers were unable to initiate flight spike patterns and displayed spontaneous bursting activity in DLMs at levels approaching that of the itpr mutants. In contrast, the fliers (three of three) retained the ability to initiate flight and showed only low levels of spontaneous spike activity, indistinguishable from the control group, WT, or Ddc-
$A$

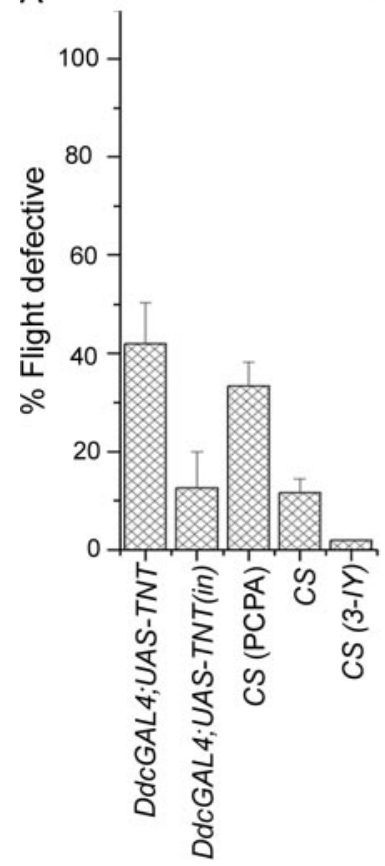

B

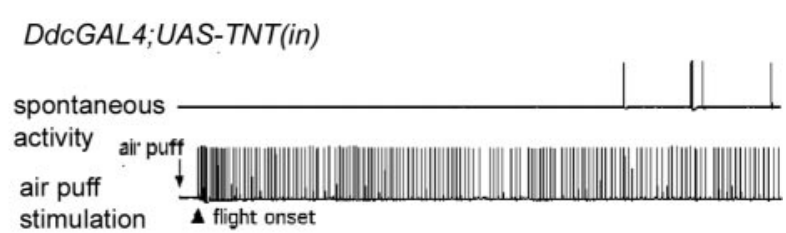

DdcGAL4;UAS-TNT (flier)

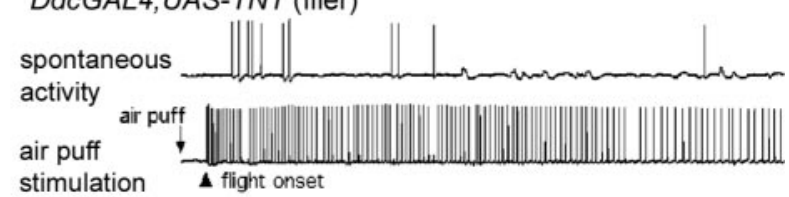

DdcGAL4;UAS-TNT (non-flier)

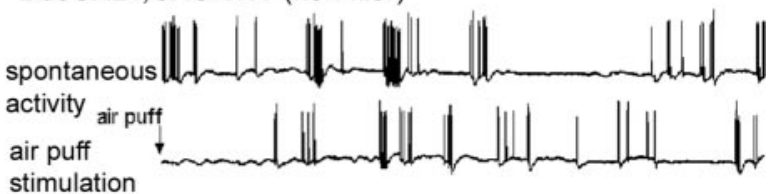

Figure 6. Abnormal flight behavior and physiology are observed after blocking synaptic transmission in DdcGAL4 neurons. $A$, Blocking synaptic transmission in DdcGAL4 (serotonin and dopamine)-positive neurons, by expression of tetanus toxin (UAS-TNT), could partially phenocopy the flight behavior defects seen in itpr mutants. Inhibition of serotonin synthesis by acute PCPA feeding of adult flies for $5 \mathrm{~d}$ also phenocopies flight defects seen in itpr mutants (see Materials and Methods and Results for details). $n \geq$ 60 for all trials. $B$, Independent samples of fliers $(n=54)$ and nonfliers $(n=160)$ were obtained from flies expressing UAS-TNT in the DdcGAL4 domain and examined for flight physiology. Flies in which an air puff failed to initiate flight activity (3 of 3 ) showed abnormally high spontaneous bursting activities in the DLMs, similar to that observed in itpr mutants. Flies that initiated flight activity on air puff stimulation (3 of 3) showed normal levels of spontaneous spiking activity. Presumably in these flies, the TNT transgene was not expressed at levels sufficient for loss of synaptic function. Normal flight activity and spontaneous firing rates were observed in control flies generated by mating flies from the DdcGal4 strain with flies carrying an inactive form of tetanus toxin, referred to as UAS-TNT(in). 
A

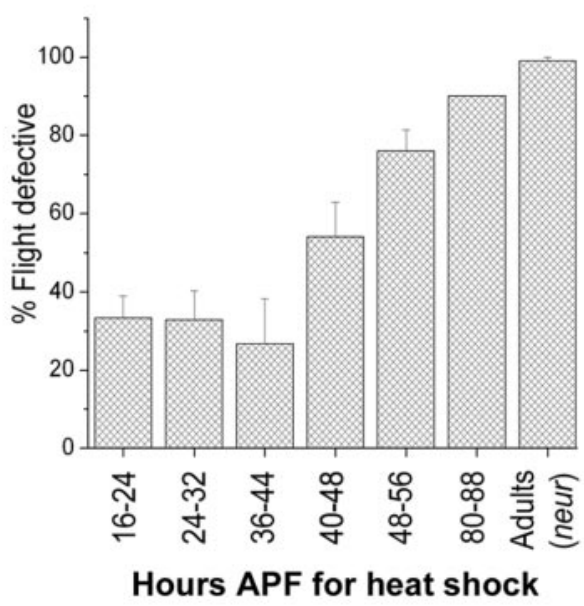

B
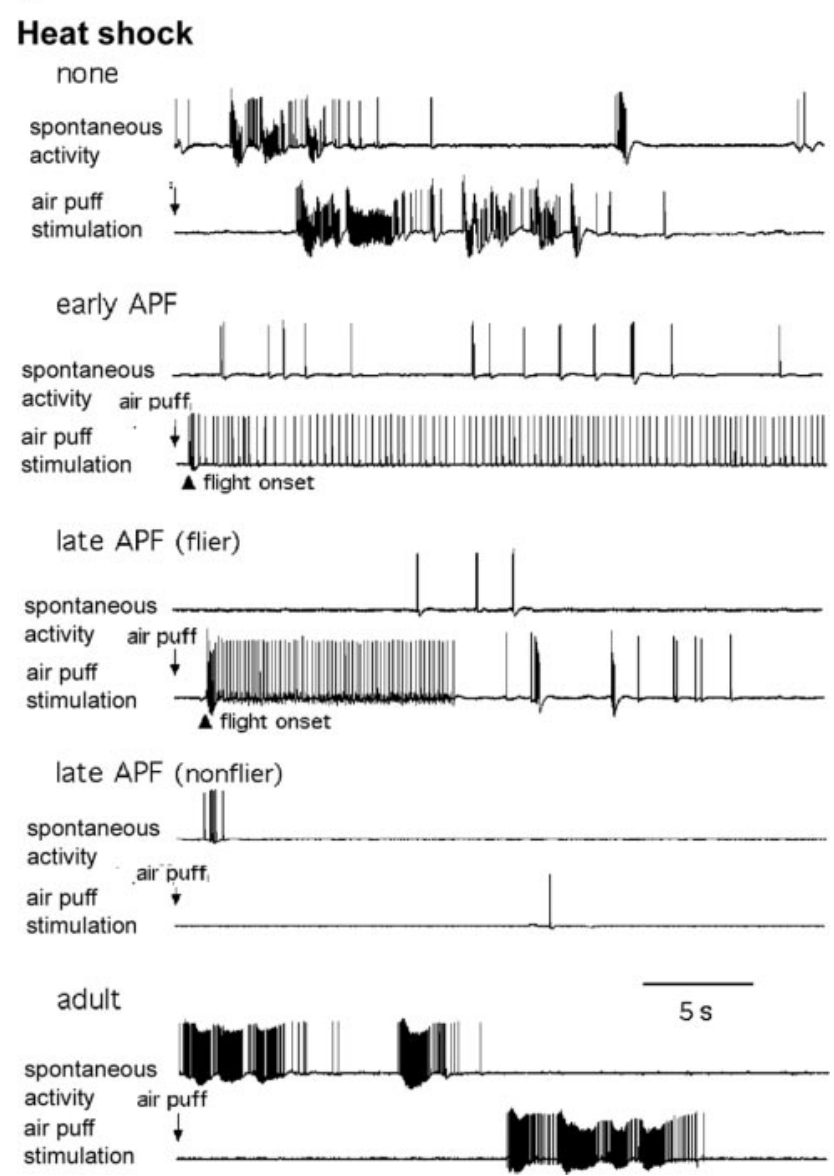

Figure 7. Expression of the itpr gene during early pupal development is sufficient to rescue flight behavior and physiology. A, Transgenic itpr ${ }^{+}$induction by a $1 \mathrm{hr}$ heat shock at $37^{\circ} \mathrm{C}$ during pupal development (at the indicated hours after puparium formation) could significantly rescue flight defects in animals of $\mathrm{UAS}^{-}-\mathrm{tpr}^{+}{ }^{+}$; $\mathrm{SGAL}^{(t) /+} ; w C 703 / \mathrm{ug} 3$ genotype. Flies were tested in batches of 10 for each time point. $n \geq 60$ for all trials. $B$, Normal spontaneous firing rates and flight activity patterns were restored in UAS-itpr ${ }^{+} ;$hSGAL $^{(t) /+}{ }^{\text {wC703/ug } 3 \text { flies after }}$ a $1 \mathrm{hr}$ heat shock of $37^{\circ} \mathrm{C}$ delivered during early pupal development (but not during adulthood). Flies obtained from pupae given a heat shock during first $2 \mathrm{~d}$ of pupal development at $25^{\circ} \mathrm{C}(5$ of 5) displayed normal levels of spontaneous DLM spike activities and the typical flight spike pattern initiated by air puffs (early APF). In contrast, only two of four flies from pupae receiving a heat shock in the last $2 \mathrm{~d}$ of pupal development displayed normal levels of spontaneous DLM spike activities and the typical flight spike pattern initiated by air puffs (late APF-flier). Late-APF nonfliers did not initiate flight activity, although the abnormal spontaneous bursting was reduced in DLMs. Adult heat shock was ineffective and still showed abnormally high spontaneous bursting activity indistinguishable from that observed in control flies without heat shock. Flies were raised at $25^{\circ} \mathrm{C}$.
GAL4; UAS-TNT(in) (Fig. 6B). Interestingly, although wing posture could be rescued by UAS-itpr ${ }^{+}$expression in DdcGAL4 cells, TNT expression in these cells does not induce abnormal wing posture (data not shown). Thus, loss of synaptic transmission in DdCGAL4 cells does not lead to the aberrant wing posture observed in itpr mutants. From these observations, there appears to be multiple roles for the itpr gene in aminergic cells during pupal development and in adulthood.

The itpr gene is necessary for development of the flight circuit in pupae

To measure the contribution of the itpr gene on development of the flight circuit during pupation, a second $h s G A L 4$ line, referred to as $h s G A L 4^{t}$, was used. This strain, unlike the strain used in the previous experiments in Figures 1-3, has no detectable basal expression at $25^{\circ} \mathrm{C}$. Flies of the genotype $U A S$-itpr/+; $h s G A L 4^{t} /+$; itpr ${ }^{w c 703} /$ itpr $^{u g 3}$, grown at $25^{\circ} \mathrm{C}$ throughout development, were indistinguishable from itpr $r^{w c 703} /$ itpr $^{\text {ug } 3}$ mutants (data not shown). Heat shocks for $1 \mathrm{hr}$ at $37^{\circ} \mathrm{C}$ were given to UAS-itpr/+; $h s G A L 4^{t} /+$; itpr $r^{w c 703} /$ itpr $^{u g 3}$ pupae during specific stages APF. These stages ranged from 16 to $88 \mathrm{hr}$ APF (Fig. 7A). The heatshocked organisms were put back at $25^{\circ} \mathrm{C}$ and tested for flight $2-3$ $\mathrm{d}$ after eclosion. Although a $1 \mathrm{hr}$ heat shock given between 24 and $48 \mathrm{hr}$ APF rescued flight to significant levels ( $~ 70 \%)$, the rescue dropped consistently when heat shocks were given after $48 \mathrm{hr}$ APF (Fig. 7A). Thus, the Ins $\mathrm{P}_{3} \mathrm{R}$ is essential for adult flight between 16 and $48 \mathrm{hr}$ APF. Together with the rescue of flight by expression of UAS-itpr ${ }^{+}$in DdCGAL4 cells, it is reasonable to assume that the requirement between 24 and $48 \mathrm{hr}$ APF is in the DdCGAL4 domain. In addition, the observation of partial loss of flight and associated physiology in DdCGAL4/UAS-TNT flies suggests that there is a requirement for DdCGAL4 neurons in the acute control of flight in adult Drosophila. This idea is supported by studies in Manduca sexta where biogenic amines have been shown to play a role in initiating, maintaining, and terminating flight behavior (Claassen and Kammer, 1986). At this stage, our experiments cannot directly address the requirement for the Ins $\mathrm{P}_{3} \mathrm{R}$ in adult DdCGAL4 neurons.

Independent electrophysiological examinations confirmed that the efficiency of rescue by expression of $U A S-i t p r^{+}$by $h s G A L 4^{t}$ varied at different developmental stages (Fig. $7 B$ ). Heat shocks delivered during the first $2 \mathrm{~d}$ of the pupal stage generated mostly fliers. Among the flies sampled for electrophysiological recordings, all (five of five) displayed normal flight patterns and low levels of spontaneous spike activity (shown as early APF in Fig. $7 B$ ). In contrast, among individuals sampled from the flies with heat shock treatment during the last $2 \mathrm{~d}$ of pupation, only two of four were able to initiate flight pattern after air puff stimulation and showed wild-type levels of spontaneous activities in DLMs (shown as late APF flier in Fig. 7B). However, DLM spike patterns reflecting flight activity could not be evoked in the rest of the flies, although they showed a level of spontaneous spike activity lower than typical itpr mutants (shown as late APF nonflier in Fig. $7 B$ ). Significantly, heat shock delivered after adult emergence was ineffective in rescuing flight, producing no flight activity pattern but a high level of spontaneous bursting activity, a phenotype indistinguishable from that of control flies without heat shock treatment (shown as adult in Fig. 7B). Nevertheless, a requirement of persistent expression of $\operatorname{Ins}_{3} \mathrm{R}$ in adult neurons remains a possibility, based on an independent observation that a GFP-tagged InsP $\mathrm{P}_{3} \mathrm{R}$ is perdurant in adults after a $1 \mathrm{hr}$ pupal heat shock given in the first $24 \mathrm{hr}$ of pupal development (data not shown). Thus, adults that eclose from pupae in 
which the $\mathrm{InsP}_{3} \mathrm{R}$ was induced between 16 and $48 \mathrm{hr}$ APF may well have sufficient Ins $\mathrm{P}_{3} \mathrm{R}$ for acute adult functions.

\section{Involvement of serotonergic interneurons in adult flight}

To determine independently which of either dopamine or serotonin neurons had a greater effect on acute activity related to adult flight, we performed the following experiments. Adult wild-type flies were fed with inhibitors of serotonin (PCPA) or dopamine (3-IY) synthesis and subsequently tested for flight. These compounds have been shown to decrease levels of serotonin (PCPA) or dopamine (3-IY) in Drosophila adults (Baier et al., 2002). Approximately $33 \%$ of adults were unable to fly after being fed with PCPA for $5 \mathrm{~d}$. However, feeding of 3-IY had no effect on flight (Fig. 6A). PCPA feeding of adult flies did not affect general locomotion as observed in a climbing test (data not shown).

Induction of flight defects by acute PCPA treatment led us to search for anatomical clues that are indicative of serotonin innervation in neural circuits involved in flight. We used the OK6GAL4 line to drive UAS-GFP in motor neurons. Adult thoracic ganglia of these animals were stained for 5-HT immunoreactivity. Complex networks of 5-HT-positive varicosities were observed in thoracic ganglia, and some of these varicosities were found to lie in the same confocal plane $(0.3 \mu \mathrm{M})$ as the cell bodies of flight motor neurons (Fig. $8 F$ ), suggesting the presence of axosomatic serotonergic synapses. These varicosities could arise from axon tracts that originate from cell bodies that lie in the brain rather than in the thoracic ganglion (Fig. 8G-I). Cell bodies of the flight motor neurons innervating the DLMs were identified by their position and axonal projections, as described by Ikeda and Koenig (1988b).

\section{Discussion}

In this work, through analysis of the flight behavior and physiology of a set of viable itpr mutant alleles, we show for the first time that the Ins $\mathrm{P}_{3} \mathrm{R}$ is required for air puff-induced flight and associated neuronal rhythmicity. This requirement is in the domain of DdCGAL4 expression during the first $48 \mathrm{hr}$ of pupal development. From pharmacological and anatomical experiments, it appears likely that the $\mathrm{InsP}_{3} \mathrm{R}$ is required in serotonergic neurons, although a requirement in dopaminergic cells cannot be ruled out at this stage.

\section{Developmental effects of itpr mutants on the function of aminergic cells}

Changes in intracellular calcium levels as a consequence of Ins $\mathrm{P}_{3} \mathrm{R}$ function are likely to affect diverse aspects of neuronal physiology. The itpr alleles studied here are viable and hence their behavioral and physiological phenotypes probably reflect neuronal functions most sensitive to $\mathrm{InsP}_{3}$-mediated $\mathrm{Ca}^{2+}$ release. In- terestingly, the foci of these functions lie in aminergic cells that release the neurotransmitters serotonin or dopamine. Furthermore, despite the fact that the observed phenotypes are behavioral and physiological, a significant proportion of these arise from a developmental requirement for the $\mathrm{Ins}_{3} \mathrm{R}$. Thus, normal formation and functioning of the air puff-stimulated flight circuit in adults requires the itpr gene during its formation and growth in pupae.

It is known that motoneurons innervating the indirect flight muscles undergo dendritic and axonal remodeling during pupariation (Consoulas et al., 2002). From several studies in both invertebrates and vertebrates, it appears that among the biogenic amines, serotonin can modulate axon outgrowth. In serotoninpositive cerebral giant cells of Lymnaea stagnalis, release of 5-HT autoregulates axon growth by inducing growth cone collapse. The cerebral giant cells form part of the neural circuit that controls rhythmic feeding behavior in these molluscs (Koert et al., 2001). In Drosophila, inhibition of serotonin synthesis has been shown to cause excessive branching of serotonergic axon terminals during embryonic and larval development (Budnik et al., 1989). More recently, serotonin has been shown to affect development of the swimming circuit in zebrafish (Brustein et al., 2003), and a role for serotonergic interneurons has been described during development of the left-right coordination of 
rhythmic motor activity in rat spinal cord (Nakayama et al., 2002). Together with these studies, it is reasonable to postulate that itpr mutants affect development of the flight circuit through modulating serotonin release. The observation that the flight deficit in DdCGAL4/UAS-TNT organisms $(\sim 45 \%)$ is less than that of a majority of the itpr mutants $(>80 \%)$ suggests that a component of this release is neurohormonal and not entirely because of evoked neuronal activity. A role for the Ins $\mathrm{P}_{3} \mathrm{R}$ in neurohormonal secretion is also apparent in DdCGAL4-positive neurons and neurohemal cells of second instar Drosophila larvae (Joshi et al., 2004). These data raise the possibility that release of serotonin is particularly sensitive to intracellular $\mathrm{Ca}^{2+}$ levels regulated through the $\operatorname{Ins}_{3} \mathrm{R}$. This idea needs to be tested rigorously by additional experiments.

\section{Ins $P_{3} R$ function in adult flight}

Both serotonin and dopamine have been shown to modulate the acute activity of motor circuits (Cooper and Neckameyer, 1999; Strawn et al., 2000; Svensson et al., 2001; Friggi-Grelin et al., 2003). Increased serotonin release has been shown to increase the postsynaptic response of flight motoneurons in locust (Parker, 1995). Recently, serotonin was shown to alter motoneuron firing in Drosophila larvae in a biphasic manner (Dasari and Cooper, 2004). The increased frequency of spontaneous firing and the loss of air puff induced flight in itpr mutants suggest that disruption of Ins $\mathrm{P}_{3} \mathrm{R}$ function could alter normal firing of motor neurons, which innervate the DLMs. The required $\mathrm{Ins}_{3} \mathrm{R}$ activity does not reside in the motor neurons but rather in aminergic (possibly serotonergic) neurons innervating and controlling motor neuron activity. One explanation is that inhibitory connections that modulate flight motor neurons have been lost or weakened during development. However, as adult feeding of PCPA results in lowered synthesis of serotonin and resembles the blocking effects of release of serotonin by TNT from these interneurons and also partially phenocopies itpr phenotype, it is possible that the Ins $\mathrm{P}_{3} \mathrm{R}$ continues to play a central role in flight coordination.

Anatomical analysis showed that cell bodies positive for serotonin and DdcGAL4 exist in the thoracic ganglia, but we failed to detect any arborization from these cell bodies toward flight motoneurons. Instead, our data suggest that 5-HT-positive varicosities on flight motor neurons arise from serotonin and DdcGAL4positive axon tracts that descend from the brain. This observation argues for a serotonergic modulation of flight motor neuron function from neurons in the brain. Localization of the $\operatorname{Ins}_{3} \mathrm{R}$ to these neurons and their axon tracts was not feasible, because immunohistochemistry with existing antisera to the Drosophila Ins $\mathrm{P}_{3} \mathrm{R}$ gave a cross-reaction with nonspecific antigen(s) in adult CNS preparations. The existing itprGAL4 line (Venkatesh et al., 2001) could not be used either, because it represents a subset of the itpr gene expression domain and does not express in the adult nervous system. Very likely, the physiological defects observed are attributable to a combination of inappropriate circuit formation during development and altered neuronal activity in adults. Although development has a significant contribution, the existing tools do not allow us to investigate an adult-specific requirement in an unambiguous manner. However, given the perdurance of the $\operatorname{Ins}_{3} \mathrm{R}$ in adults (as judged by the expression of a $U A S$-itprGFP transgene induced in pupae by the same heat shock regime as that for $U A S-i t p r^{+}$), a contribution to acute flight signaling remains a distinct possibility.

Our studies show that the $\mathrm{Ins}_{3} \mathrm{R}$ is essential for development of the neural circuit that probably functions as the central pattern generator for air puff-induced flight. The function of the $\operatorname{Ins}_{3} \mathrm{R}$ is in aminergic cells, indicating that at a cellular level, it functions in the release of serotonin and/or dopamine. Earlier, $\mathrm{Ca}^{2+}$ release from vesicular InsP $\mathrm{P}_{3} \mathrm{Rs}$ in chromaffin cells, acinar cells, and islet cells have been proposed to release $\mathrm{Ca}^{2+}$ required for secretion (Vermassen et al., 2004). Its profound developmental and functional actions through release of biogenic amines is a novel finding and could have relevance for vertebrate systems, where serotonin has been shown to affect neural circuit development. Significantly, knock-outs for the $\operatorname{Ins}_{3} \mathrm{R} 1$ in mice exhibit ataxia and motor discoordination at birth (Matsumoto et al., 1996; Ogura et al., 2001), for which the underlying cellular malfunctioning is unclear. Our results should allow for a better understanding of the general principles governing invertebrate and vertebrate motor coordination.

\section{References}

Aberle H, Haghighi AP, Fetter RD, McCabe BD, Magalhaes TR, Goodman CS (2002) Wishful thinking encodes a BMP type II receptor that regulates synaptic growth in Drosophila. Neuron 33:545-558.

Acharya JK, Jalink K, Hardy RW, Hartenstein V, Zuker CS (1997) InsP3 receptor is essential for growth and differentiation but not for vision in Drosophila. Neuron 18:881-887.

Baier A, Wittek B, Brembs B (2002) Drosophila as a new model organism for the neurobiology of aggression? J Exp Biol 205:1233-1240.

Bainton RJ, Tsai LT, Singh CM, Moore MS, Neckameyer WS, Heberlein U (2000) Dopamine modulates acute responses to cocaine, nicotine and ethanol in Drosophila. Curr Biol 10:187-194.

Benzer S (1973) Genetic dissection of behavior. Sci Am 229:24-37.

Berridge MJ (1993) Inositol trisphosphate and calcium signalling. Nature 361:315-325.

Berridge MJ (1998) Neuronal calcium signaling. Neuron 21:13-26.

Brustein E, Chong M, Holmqvist B, Drapeau P (2003) Serotonin patterns locomotor network activity in the developing zebrafish by modulating quiescent periods. J Neurobiol 57:303-322.

Budnik V, Wu CF, White K (1989) Altered branching of serotonincontaining neurons in Drosophila mutants unable to synthesize serotonin and dopamine. J Neurosci 9:2866-2877.

Burg MG, Wu CF (1986) Differentiation and central projections of peripheral sensory cells with action-potential block in Drosophila mosaics. J Neurosci 6:2968-2976.

Claassen DE, Kammer AE (1986) Effects of octopamine, dopamine, and serotonin on production of flight motor output by thoracic ganglia of Manduca sexta. J Neurobiol 17:1-14.

Clandinin TR, DeModena JA, Sternberg PW (1998) Inositol trisphosphate mediates a RAS-independent response to LET-23 receptor tyrosine kinase activation in C. elegans. Cell 92:523-533.

Consoulas C, Restifo LL, Levine RB (2002) Dendritic remodeling and growth of motoneurons during metamorphosis of Drosophila melanogaster. J Neurosci 22:4906-4917.

Cooper RL, Neckameyer WS (1999) Dopaminergic modulation of motor neuron activity and neuromuscular function in Drosophila melanogaster. Comp Biochem Physiol B Biochem Mol Biol 122:199-210.

Dal Santo P, Logan MA, Chisholm AD, Jorgensen EM (1999) The inositol trisphosphate receptor regulates a 50-second behavioral rhythm in C. elegans. Cell 98:757-767.

Dasari S, Cooper RL (2004) Modulation of sensory-CNS-motor circuits by serotonin, octopamine, and dopamine in semi-intact Drosophila larva. Neurosci Res 48:221-227.

Deshpande M, Venkatesh K, Rodrigues V, Hasan G (2000) The inositol 1,4,5-trisphosphate receptor is required for maintenance of olfactory adaptation in Drosophila antennae. J Neurobiol 43:282-288.

DeSimone SM, White K (1993) The Drosophila erect wing gene, which is important for both neuronal and muscle development, encodes a protein which is similar to the sea urchin P3A2 DNA binding protein. Mol Cell Biol 13:3641-3649.

Engel JE, Wu CF (1992) Interactions of membrane excitability mutations affecting potassium and sodium currents in the flight and giant fiber escape systems of Drosophila. J Comp Physiol [A] 171:93-104.

Engel JE, Wu CF (1996) Altered habituation of an identified escape circuit in Drosophila memory mutants. J Neurosci 16:3486-3499. 
Fleming RJ, DeSimone SM, White K (1989) Molecular isolation and analysis of the erect wing locus in Drosophila melanogaster. Mol Cell Biol 9:719-725.

Friggi-Grelin F, Coulom H, Meller M, Gomez D, Hirsh J, Birman S (2003) Targeted gene expression in Drosophila dopaminergic cells using regulatory sequences from tyrosine hydroxylase. J Neurobiol 54:618-627.

Greene JC, Whitworth AJ, Kuo I, Andrews LA, Feany MB, Pallanck LJ (2003) Mitochondrial pathology and apoptotic muscle degeneration in Drosophila parkin mutants. Proc Natl Acad Sci USA 100:4078-4083.

Greeve I, Kretzschmar D, Tschape JA, Beyn A, Brellinger C, Schweizer M, Nitsch RM, Reifegerste R (2004) Age-dependent neurodegeneration and Alzheimer-amyloid plaque formation in transgenic Drosophila. J Neurosci 24:3899-3906.

Huang Y, Stern M (2002) In vivo properties of the Drosophila inebriatedencoded neurotransmitter transporter. J Neurosci 22:1698-1708.

Ikeda K, Koenig JH (1988a) Spontaneous release of multiquantal miniature excitatory junction potentials induced by a Drosophila mutant. J Physiol (Lond) 406:215-223.

Ikeda K, Koenig JH (1988b) Morphological identification of the motor neurons innervating the dorsal longitudinal flight muscle of Drosophila melanogaster. J Comp Neurol 273:436-444.

Jacobs K, Todman MG, Allen MJ, Davies JA, Bacon JP (2000) Synaptogenesis in the giant-fibre system of Drosophila: interaction of the giant fibre and its major motorneuronal target. Development 127:5203-5212.

Jhaveri D, Sen A, Reddy GV, Rodrigues V (2000) Sense organ identity in the Drosophila antenna is specified by the expression of the proneural gene atonal. Mech Dev 99:101-111.

Joshi R, Venkatesh K, Srinivas R, Nair S, Hasan G (2004) Genetic dissection of itpr gene function reveals a vital requirement in aminergic cells of Drosophila larvae. Genetics 166:225-236.

Koert CE, Spencer GE, van Minnen J, Li KW, Geraerts WP, Syed NI, Smit AB, van Kesteren RE (2001) Functional implications of neurotransmitter expression during axonal regeneration: serotonin, but not peptides, autoregulate axon growth of an identified central neuron. J Neurosci 21:5597-5606.

Kraminsky GP, Clark WC, Estelle MA, Gietz RD, Sage BA, O'Connor JD, Hodgetts RB (1980) Induction of translatable mRNA for dopa decarboxylase in Drosophila: an early response to ecdysterone. Proc Natl Acad Sci USA 77:4175-4179.

Lee J, Wu CF (2002) Electroconvulsive seizure behavior in Drosophila: analysis of the physiological repertoire underlying a stereotyped action pattern in bang-sensitive mutants. J Neurosci 22:11065-11079.

Li H, Chaney S, Roberts IJ, Forte M, Hirsh J (2000) Ectopic G-protein expression in dopamine and serotonin neurons blocks cocaine sensitization in Drosophila melanogaster. Curr Biol 10:211-214.

Luo L, Liao YJ, Jan LY, Jan YN (1994) Distinct morphogenetic functions of similar small GTPases: Drosophila Drac1 is involved in axonal outgrowth and myoblast fusion. Genes Dev 8:1787-1802.

Maeda N, Kawasaki T, Nakade S, Yokota N, Taguchi T, Kasai M, Mikoshiba K (1991) Structural and functional characterization of inositol 1,4,5trisphosphate receptor channel from mouse cerebellum. J Biol Chem 266:1109-1116

Matsumoto M, Nakagawa T, Inoue T, Nagata E, Tanaka K, Takano H, Minowa O, Kuno J, Sakakibara S, Yamada M, Yoneshima H, Miyawaki A, Fukuuchi Y, Furuichi T, Okano H, Mikoshiba K, Noda T (1996) Ataxia and epileptic seizures in mice lacking type 1 inositol 1,4,5-trisphosphate receptor. Nature 379:168-171.

Miyata M, Finch EA, Khiroug L, Hashimoto K, Hayasaka S, Oda SI, Inouye M, Takagishi Y, Augustine GJ, Kano M (2000) Local calcium release in dendritic spines required for long-term synaptic depression. Neuron 28:233-244.

Nakayama K, Nishimaru H, Kudo N (2002) Basis of changes in left-right coordination of rhythmic motor activity during development in the rat spinal cord. J Neurosci 22:10388-10398.

Ogura H, Matsumoto M, Mikoshiba K (2001) Motor discoordination in mutant mice heterozygous for the type 1 inositol 1,4,5-trisphosphate receptor. Behav Brain Res 122:215-219.

Parker D (1995) Serotonergic modulation of locust motor neurons. J Neurophysiol 73:923-932.

Raghu P, Colley NJ, Webel R, James T, Hasan G, Danin M, Selinger Z, Hardie RC (2000) Normal phototransduction in Drosophila photoreceptors lacking an InsP(3) receptor gene. Mol Cell Neurosci 15:429-445.

Ranganayakulu G, Schulz RA, Olson EN (1996) Wingless signaling induces nautilus expression in the ventral mesoderm of the Drosophila embryo. Dev Biol 176:143-148.

Serysheva II, Bare DJ, Ludtke SJ, Kettlun CS, Chiu W, Mignery GA (2003) Structure of the type 1 inositol 1,4,5-trisphosphate receptor revealed by electron cryomicroscopy. J Biol Chem 278:21319-21322.

Srikanth S, Wang Z, Tu H, Nair S, Mathew M, Hasan G, Bezprozvanny I (2004) Functional properties of the Drosophila melanogaster Inositol 1,4,5-trisphosphate receptor mutants. Biophysical J 86:3634-3646.

Strawn JR, Neckameyer WS, Cooper RL (2000) The effects of 5-HT on sensory, central and motor neurons driving the abdominal superficial flexor muscles in the crayfish. Comp Biochem Physiol B Biochem Mol Biol 127:533-550.

Svensson E, Grillner S, Parker D (2001) Gating and braking of short- and long-term modulatory effects by interactions between colocalized neuromodulators. J Neurosci 21:5984-5992.

Sweeney ST, Broadie K, Keane J, Niemann H, O’Kane CJ (1995) Targeted expression of tetanus toxin light chain in Drosophila specifically eliminates synaptic transmission and causes behavioral defects. Neuron 14:341-351.

Tanouye MA, Wyman RJ (1980) Motor outputs of giant nerve fiber in Drosophila. J Neurophysiol 44:405-421.

Vandervorst P, Ghysen A (1980) Genetic control of sensory connections in Drosophila. Nature 286:65-67.

Venkatesh K, Hasan G (1997) Disruption of the IP3 receptor gene of Drosophila affects larval metamorphosis and ecdysone release. Curr Biol 7:500-509.

Venkatesh K, Siddhartha G, Joshi R, Patel S, Hasan G (2001) Interactions between the inositol 1,4,5-trisphosphate and cyclic AMP signaling pathways regulate larval molting in Drosophila. Genetics 158:309-318.

Vermassen E, Parys JB, Mauger JP (2004) Subcellular distribution of the inositol 1,4,5-trisphosphate receptors: functional relevance and molecular determinants. Biol Cell 96:3-17.

Yasuyama K, Salvaterra PM (1999) Localization of choline acetyltransferase-expressing neurons in Drosophila nervous system. Microsc Res Tech 45:65-79.

Zars T, Fischer M, Schulz R, Heisenberg M (2000) Localization of a shortterm memory in Drosophila. Science 288:672-675. 